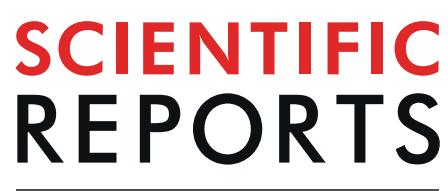

natureresearch

Check for updates

\title{
Identification of candidate chromosome region of Sbwm1 for Soil-borne wheat mosaic virus resistance in wheat
}

\author{
Shubing Liu ${ }^{1 凶}$, Guihua Bai $\mathbb{1}^{2,3 凶}$, Meng Lin ${ }^{3}$, Mingcheng Luo $\mathbb{C}^{4}$, Dadong Zhang ${ }^{3}$, Feng Jin ${ }^{3,5}$, \\ Bin Tian $\mathbb{1}^{6}$, Harold N. Trick $\mathbb{1}^{6}$ \& Liuling Yan $\mathbb{D}^{7}$
}

Soil-borne wheat mosaic virus (SBWMV) causes a serious viral disease that can significantly reduce grain yield in winter wheat worldwide. Using resistant cultivars is the only feasible strategy to reduce the losses caused by SBWMV. To fine map the resistance gene Sbwm1, 205 wheat accessions was genotyped using wheat Infinium iSelect Beadchips with $90 \mathrm{~K}$ SNPs. Association analysis identified 35 SNPs in 12 wheat genes and one intergenic SNP in the Sbwm1 region that showed a significant association with SBWMV resistance. Those SNPs were converted into Kompetitive Allele-Specific Polymerase assays (KASP) and analyzed in two $\mathrm{F}_{6}$-derived recombinant inbred line (RIL) populations derived from the crosses between two resistant cultivars 'Wesley' and 'Deliver' and a susceptible line 'OK03825-5403-6'. Linkage analysis mapped this gene on chromosome 5D at intervals of $5.1 \mathrm{cM}$ and $3.4 \mathrm{cM}$ in the two populations, respectively. The two flanking markers in both populations delimited the gene to a $620 \mathrm{~kb}$ region where 19 genes were annotated. Comparative analysis identified a syntenic region of $660 \mathrm{~kb}$ in Ae. tauschii with 18 annotated genes and a syntenic region in chromosome 1 of $B$. distachyon. The candidate region includes several disease resistance related genes and we identified a PTI1-like tyrosine-protein kinase 1 gene as a putative candidate gene for Sbwm1. The two flanking SNPs for Sbwm 1 can effectively separate the resistant and susceptible lines in a new diversity panel of 159 wheat germplasm. The results from this study lay a solid foundation for the cloning, functional characterization and marker-assisted selection of Sbwm1.

Soil-borne wheat mosaic (SBWM) disease, caused by Soil-borne wheat mosaic virus (SBWMV) was first appeared in the Great Plains of the U.S.A. in $1919^{1}$ and has become an important disease in most winter wheat growing regions globally ${ }^{2-7}$. SBWMV infects wheat roots in wet soil during germination at $15^{\circ} \mathrm{C}$ to $20^{\circ} \mathrm{C}$ with an optimum temperature at $17^{\circ} \mathrm{C}$. Soil water is critical for the swimming zoospore to reach host roots ${ }^{8,9}$. Virus movement from the site of infection on roots to the leaves occurs at temperatures lower than $20^{\circ} \mathrm{C}$. Higher temperatures limit virus movement into leaves and further infection in developing leaves will not occur if temperatures rise to more than $20^{\circ} \mathrm{C}$ after some leaves are infected ${ }^{9}$.

The SBWMV symptom usually first appears in early spring when the crop begins to green up. Low spring temperatures is usually conducive to the occurrence of SBWMV and could promote the development of the symptoms. Irregular chlorotic patches in a field indicate SBWMV infection. In dry environments, patches of symptomatic SBWMV-infected plants usually present in low-lying, wet regions of a field that are favorable for infection by the swimming zoospores of Polymyxa graminis, the vector of SBWMV ${ }^{10}$. In wet soil climates, these patches may present anywhere in an infected field. SBWMV symptoms stop developing on leaves when the average temperature exceeds $20^{\circ} \mathrm{C}$, but can be reinitiated on newly emerged leaves given conductive environmental

${ }^{1}$ State Key Laboratory of Crop Biology, College of Agronomy, Shandong Agricultural University, Taian, Shandong, 271018, China. ${ }^{2}$ USDA-ARS, Hard Winter Wheat Genetics Research Unit, 4008 Throckmorton Hall, Manhattan, KS, 66506, USA. ${ }^{3}$ Department of Agronomy, Kansas State University, Manhattan, KS, 66506, USA. ${ }^{4}$ Department of Plant Science, University of California- Davis, Davis, CA, 95616, USA. ${ }^{5}$ Chengdu Donnees Biotechnology Co., Ltd, Chengdu, Sichuan, 610000, China. ${ }^{6}$ Department of Plant Pathology, Kansas State University, Manhattan, KS, 66506, USA. ${ }^{7}$ Department of Plant and Soil Sciences, Oklahoma State University, Stillwater, OK, 74078, USA. ${ }_{\mathrm{e}}$-mail: sbliu@ sdau.edu.cn; guihua.bai@ars.usda.gov 
conditions ${ }^{10}$. Reported yield losses caused by SBWMV ranged from $10 \%$ to $30 \%$ and can reach up to $80 \%$ in SBWMV severely occured fields in the U.S.A. and 50\% in Brazil ${ }^{11-13}$.

Polymyxa graminis is one of the endoparasitic slime molds (Plasmodiophoromycota), which can produce resting spores that harbor viral RNA and movement protein, and can be easily distributed by wind, water and machinery. Those resting virus-containing spores can remain dormant and invasive in soil for up to 30 years, and then germinate in an environment favorable for infection when host are available. Thus, it is very difficult to control this disease once it happened in a field. The most practical SBWM control strategy is to grow resistant cultivars ${ }^{2,14,15}$.

Inheritance studies have shown that resistance to SBWMV is controlled by major genes. Winter wheat cultivars such as Shawnee, Centurk and KS73256 were reported to carry a dominant gene for SBWMV resistance. Newton might contain a gene for resistance different from that in 'Arthur 71', 'Homestead', and 'Tascosa'14. However, a cultivar 'Embrapa 16' from Brazil was reported to carry two genes for SBWMV resistance ${ }^{2}$. With the rapid development and wide use of molecular markers in wheat genetic studies, quantitative trait locus (QTL) mapping approach has been used to localize SBWMV resistance genes using linkage maps. The QTL on 5DL has been reported by several independent studies on winter wheat 'Karl 92'16, 'Pioneer 26R61', 'AGS 2020' and 'Heyne'12,17. A breeding line KS96WGRC40 derived from Aegilops tauschii also carries this gene ${ }^{18}$. This gene was designated as $S b w m 1^{17}$. Interestingly, a gene conferring resistance to Soil-borne cereal mosaic virus (SBCMV) in 'Cadenza, 'Tremie' and 'Claire', designated as $S b m 1$, was also mapped in the region of $S b w m 1^{19,20}$. Because they tightly linked to the marker Xgwm469-5D, Sbm1 for SBCMV resistance is either a tightly linked or the same gene as Sbwm1 for SBWMV resistance.

Single nucleotide polymorphisms (SNPs) are the most widely distributed DNA sequence variations in a genome. During the past decade, next-generation sequencing (NGS) technologies have become the cheapest and fastest technology for genome-wide SNPs discovery ${ }^{21}$. Fixed SNP chips have been released and widely used for various genetic and breeding studies including genome-wide association analysis and genomic selection in many crop species ${ }^{21-23}$. In wheat, SNP chips with various number of SNPs including Wheat $9 \mathrm{~K}$ iSelect, Wheat $90 \mathrm{~K}$ iSelect and Wheat $660 \mathrm{~K}$ Axiom SNP chips have been developed and widely used to map genes or QTLs for different traits ${ }^{24-28}$.

In a former study, a SSR marker Xgwm469, that was associated with the SBWMV resistance on chromosome $5 \mathrm{DL}$ has been identified ${ }^{29}$. Further association mapping analysis using Wheat $9 \mathrm{~K}$ iSelect chip identified six SNPs from two genes that were highly associated with SBWMV resistance, and the identified SNPs were further mapped on 5DL using a F6 RIL population 'Heyne'/'Trego' ${ }^{17}$. However, those SNPs were mapped on one side of $S b w m 1$ and the marker flanking on the other side of $S b w m 1$ is about $20 \mathrm{cM}$ a way. Therefore, a tightly linked flanking marker is urgently needed for effective marker-assisted transfer of Sbwm 1 to new wheat cultivars and cloning Sbwm1.

In this study, the panel of 205 wheat accessions previously genotyped with $9 \mathrm{~K}$ SNP chips were further genotyped using Infinium $90 \mathrm{~K}$ wheat SNP chips to identify more flanking SNPs for Sbwm1. Two new RIL populations were used to map the SBWMV resistance genes in the two resistant cultivars Wesley and Deliver and to verify the linkage among the newly identified SNP markers Sbwm1. We also pinpointed the candidate region for Sbwm1 using the Chinese Spring reference genome, developed KASP assays for the linked SNPs to Sbwm1 for marker-assisted selection (MAS), and identified a putative candidate gene for $S b w m 1$ by gene expression analysis.

\section{Materials and Methods}

Plant materials. The association mapping panel includes 205 wheat accessions with 137 hard winter wheat (HWW) and 68 soft winter wheat (SWW) from six 2008 HWW and SWW nurseries (Supplementary Table S1). Seeds for DNA isolation and disease evaluation were originated from a single plant of each accession increased in a greenhouse to minimize within-line heterogeneity ${ }^{29}$. All these accessions were genotyped using Infinium iSelect $90 \mathrm{~K}$ wheat SNP chips ${ }^{25}$. A second panel of 159 wheat cultivars and breeding lines were used to validate the mapped KASP assays (Supplementary Table S2).

Two $\mathrm{F}_{6}$ RIL populations developed by single-seed descent from the crosses 'Wesley' x OK03825-5403-6 (180 RILs) and 'Deliver' $x$ OK03825-5403-6 (260 RILs) were used to map the SBWMV resistance gene. 'Wesley' and 'Deliver' are SBWMV-resistant HWW cultivars, whereas OK03825-5403-6 is a highly SBWMV-susceptible HWW breeding line.

Disease evaluation. The association panel of 205 wheat accessions were evaluated for SBWMV resistance in the SBWMV-infested field at the Rocky Ford Research Farm of Kansas State University, Manhattan, KS in 20092010 and 2010-2011 wheat growing seasons. The nursery has shown consistent and severe SBWMV infection on susceptible wheat cultivars since 2006. Field experimental design, planting, and disease damage evaluation followed Zhang et al..$^{30}$. An additional panel of 159 accessions were evaluated for SBWMV resistance in 2010-2011 and 2011-2012 wheat growing seasons in the same field nursery for marker validation. The two RIL populations were evaluated for SBWMV resistance in the same field in 2013-2014 and 2014-2015 growing seasons using the same rating scale described for association mapping. All experiments used random complete block design with two replicates. In all experiments, each accession or RIL was sowed at $1.5 \mathrm{~m}$-long rows with planting density of 40 seeds. After planting, the nursery was sprinkler irrigated everyday for 7 days till the seedling emergence to maintain high moisture in the soil to promote SBWMV infection.

DNA extraction and $90 \mathrm{~K}$ SNP assay. The 205 accessions were genotyped using the $90 \mathrm{~K}$ SNP chips at USDA-ARS Cereal Crops Research Unit, Fargo, ND as described by Wang et al. ${ }^{25}$. SNPs with less than $5 \%$ minor allele frequency (MAF) or with more than $15 \%$ missing data were removed ${ }^{31}$. A total of 21,600 SNPs were scored and used in the final analysis. Sequences that harbored significant SNPs were blasted against the Chinese Spring 


\begin{tabular}{|l|l|l|}
\hline SNP Name & Left Primer & Right Primers \\
\hline BS00067308_51-FAM & ATGCTGCATCCACGTCCTt & CACGTCTTCACGGATGTTTTT \\
\hline BS00067308_51-HEX & ATGCTGCATCCACGTCCTg & \\
\hline CAP12_c5949_104-FAM & GCATCAGGAAGAGGAACAGCa & GCCGTCGTAGTAGGTGAAGG \\
\hline CAP12_c5949_104-HEX & GCATCAGGAAGAGGAACAGCc & \\
\hline BS00079676_51-FAM & GGGTACTCTCGTCTTCCTGCATa & CAGTACAAAGCGCAACCTCA \\
\hline BS00079676_51-HEX & GGGTACTCTCGTCTTCCTGCATg & \\
\hline Kukri_c5528_603-FAM & CCAGTTAAAGGCTACATGGAGAt & AGGCCACATGAATGAGATCC \\
\hline Kukri_c5528_603-HEX & CCAGTTAAAGGCTACATGGAGAc & \\
\hline BS00011469_51-FAM & TGACTGGAAGGAAACCGGTt & GCCCAAGTGACTAAACTTTGC \\
\hline BS00011469_51-HEX & TGACTGGAAGGAAACCGGTc & \\
\hline BS00073116_51-FAM & CCGTGTGATAAGGATTCTGGt & GTTGTGTGGAGCACAGCACT \\
\hline BS00073116_51-HEX & CCGTGTGATAAGGATTCTGGg & \\
\hline BS00105939_51-FAM & GGTTGAGCAGGAAGCAGGa & GCAAGCAGACCAGTCTCTCA \\
\hline BS00105939_51-HEX & GGTTGAGCAGGAAGCAGGg & \\
\hline Contig08110-FAM & CCACCAATGGACACAAGGc & TCATCGCTCCAGGAATTACA \\
\hline Contig08110-HEX & CCACCAATGGACACAAGGt & \\
\hline Excalibur_c22724_85-FAM & ACCATTCACAACAATTGCGt & GATAATTTGTGCCGCGAGTT \\
\hline Excalibur_c22724_85-HEX & ACCATTCACAACAATTGCGc & \\
\hline Excalibur_c2795_1518-FAM & ATATACAAGGTATATTAGGt & TAACCCACCACCTGGCTTCT \\
\hline Excalibur_c2795_1518-HEX & ATATACAAGGTATATTAGGc & \\
\hline BobWhite_c13030_406-FAM & ATGCTCCCGGGCTTGATa & CCCATCACGCTCGACTTC \\
\hline BobWhite_c13030_406-HEX & ATGCTCCCGGGCTTGATg & \\
\hline
\end{tabular}

Table 1. Primers for KASP-SNPs that are highly associated with SBWMV resistance in the association mapping population.

Wheat SeqVer1.0 reference sequence ${ }^{32}$ to assign their putative chromosome positions and were converted to Kompetitive Allele Specific Polymerase Chain Reaction (KASP) assays for screening the two RIL populations (Table 1). KASP analysis following the methods described by Liu et al. ${ }^{17}$.

Development of SNP markers using Ae. tauschii reference genome. To identify more markers in the SBWMV resistance QTL region, the probe sequences harboring the identified SNPs for SBWMV resistance were used to blast the draft Ae. tauschii reference genome ${ }^{33}$. Fourteen mapped SNPs (listed in Supplementary Table S4) in Ae. tauschii physical map in the QTL region were identified and converted to KASP assays to screen the three parents, Wesley, Deliver and OK03825-5403-6, of the two RIL populations, and the polymorphic SNP (Contig08110) was screened across the two RIL populations (Table 1).

Statistical analysis. Data for SBWMV phenotypes were analyzed using two-way GLM in SAS for Windows v9 (SAS Institute, Cary, NC) to determine the effects of genotypes (g), environments (e), and G x E interactions. Heritability $\left(\mathrm{H}^{2}\right)$ was estimated using the formula

$$
\mathrm{H}^{2}=6_{\mathrm{g}}^{2} /\left(6_{\mathrm{g}}^{2}+6_{\mathrm{ge}}^{2} / \mathrm{n}+6_{\mathrm{e}}^{2} / \mathrm{nr}\right)
$$

where $\sigma_{\mathrm{g}}{ }^{2}$ is the variance among RILs, $\sigma_{\mathrm{ge}}{ }^{2}$ is the variance for $\mathrm{Gx} \mathrm{E} ; \sigma_{\mathrm{e}}{ }^{2}$ is the variance of environments, $\mathrm{n}$ is the number of environments, and $\mathrm{r}$ is the number of replicates ${ }^{34}$.

Population structure, kinship, linkage disequilibrium and association analysis of the AM Panel. Population structure was assessed by Structure 2.3.4 $4^{35}$ using a set of 1,500 SNPs that are evenly distributed on all the 21 wheat chromosomes ${ }^{31}$. The admixture model was used for structure analysis and the number of sub-populations $(\mathrm{K})$ was set as 1 to 10 with variable length of burn-in period and number of iterations at 20,000-250,000. For each trait, Bayesian information criterion (BIC) was applied to determine the optimum number of subpopulations ${ }^{31}$. Kinship was calculated using the same set of 1,500 SNPs used for structure analysis and SPAGeDi package ${ }^{36}$.

Genome-wide association analysis used the generalized linear model (GLM) with the Q matrix as fixed effects and the mixed linear model (MLM) with a Q matrix as fixed effects and a kinship matrix as random effects. The models were applied to each experiment for SBWMV resistance, and model fitness was determined based on the Bayesian information criterion value.

Association analysis of SNP data was conducted using the genome association and prediction integrated tool (GAPIT) implemented in a R package ${ }^{37}$. A threshold of $p<10^{-5}$ (1/number of markers) was set to claim significant marker-trait associations. Linkage disequilibrium and haplotype analyses of the significant SNPs were performed using HAPLOVIEW v.4.2 (http://www.broadinstitute.org/scientific-community/science/programs/ medical-and-population-genetics/haploview/haploview). 
Linkage mapping. Genotypes with disease rating of 1-2 were classified as resistant and 3-4 as susceptible for SBWMV resistance in the two RIL populations. SNP linkage maps were constructed for the two RIL populations using JoinMap ver. $4.0^{38}$. Recombination fractions were converted into centiMorgans (cM) using the Kosambi function ${ }^{39}$. The threshold of logarithm of odd (LOD) score was set at 3.0 to claim linkage among markers with 0.4 as a maximum fraction of recombination. The goodness-of-fit between observed and expected segregation ratios between two alleles was analyzed for each marker locus using a chi-square-test.

Marker sequence analysis and candidate gene annotation. Sequences that contain significant SNPs associated with SBWMV resistance were used as queries to blast the IWGSC Chinese Spring reference genome sequence RefSeqv1.0, Ae. tauschii reference genome sequence and B. distachyon genome sequence using BLASTN $^{32,40,41}$. A significant match was declared when the queried sequences showed at least $99 \%$ nucleotide identity with an e-value lower than $\mathrm{e}^{-20}$.

RNA extraction and gene expression analysis. Leaf tissues were collected from seedlings of Wesley and OK03825-5403-6 grown in the SBWMV field nursery for RNA extraction in fall 2015 and spring 2016 when the SBWMV symptom appeared. RNA was extracted and purified using the RNeasy Plant Kit with on-column DNaseI treatment (Qiagen, Valencia, CA). Complementary DNA from reverse-transcription reaction using a SuperScriptRII kit (Invitrogen, Grand Island, NY) was amplified by conventional reverse transcriptase-PCR (RT-PCR). The annotated genes in the target region were tested for the gene expression using semi-quantitative PCR analysis. Each PCR reaction mixture consisted of $15.5 \mu \mathrm{L} \mathrm{H}_{2} \mathrm{O}, 2.4 \mu \mathrm{L} 10 \times$ buffer, $2.4 \mu \mathrm{L} 50 \mathrm{mM} \mathrm{MgCl} 2,1 \mu \mathrm{L}$ of $10 \mathrm{mM}$ dNTPs, $1 \mu \mathrm{L}$ each primer at $20 \mu \mathrm{M}, 2.5 \mathrm{U}$ Taq polymerase, and $1 \mu \mathrm{L}$ cDNA. Cycle conditions were $95^{\circ} \mathrm{C}$ for $1 \mathrm{~min}$, annealing at $55-60^{\circ} \mathrm{C}$ for $1 \mathrm{~min}$ for different primers, and extension at $72^{\circ} \mathrm{C}$ for $1 \mathrm{~min}$. A final extension at $72^{\circ} \mathrm{C}$ was performed for $10 \mathrm{~min}$. Annealing temperatures and cycle conditions for each primer pair were optimized such that each PCR was in the linear range of amplification. PCR primers were listed in Supplementary Table S5.

The identified candidate gene TraesCS5D01G531200 was further quantified with three replications by quantitative real-time PCR performed on an ABI7900HT instrument using SYBR Green (Thermo Fisher Scientific). Gene expression level was analyzed using the $2^{-\Delta \Delta C t}$ method. Actin gene was used as the endogenous control with ACCTTCAGTTGCCCAGCAAT as the forward primer, CAGAGTCGAGCACAATACCAGTTG as the reverse primer.

\section{Results}

SBWMV resistance in the association and linkage mapping populations. High correlation coefficients were observed for SBWMV resistance between the two years of phenotypic data from the marker validation population $(\mathrm{r}=0.92, p<0.01)$, the two RIL populations $(\mathrm{r}=0.81$ and $0.94, p<0.01)$, and the association mapping population $(\mathrm{r}=0.85, p<0.01)^{17}$, suggesting a high repeatability of SBWMV resistance data between the field experiments. Analysis of variance showed highly significant genotypic effects and very high heritabilities (from $84.9 \%$ to $98.2 \%$ ) in both the RIL populations and the validation population (Supplementary Table S3). In the two biparental populations, the distribution of SBWMV disease scores deviated significantly from a normal distribution and showed an obvious bimodal distribution with two peaks towards the resistant and susceptible parents (Fig. 1), suggesting that a major gene is responsible for SBWMV resistance in Wesley and Deliver.

Association analysis identified SNPs for the SBWMV resistance. Genotyping the association mapping population with the $90 \mathrm{~K}$ SNP chips identified 21,600 polymorphic SNPs. Using the MLM model, thirty-five SNPs were identified significantly associated with SBWMV resistance in both years $\left(P<10^{-5}\right)$ (Fig. 2, Table 2), with 33 of them from 11 annotated high confidence genes (from TraesCS5D01G529700 to TraesCS5D01G532100) on chromosome 5D. SNP Excalibur_c22724_85 was from an annotated low confidence gene TraesCS5D01G625100LC, and BS00013935_51 was from an intergenic region. Using the Chinese Spring reference genome (IWGSC RefSeq v1.0), those SNPs can be located in a $69 \mathrm{Mb}$ interval on 5D. However, when SNP BS00013935_51, the SNP with the lowest association among those SNPs $(P=1.44 E-07)$ was excluded, the interval can be delimited to a $1.18 \mathrm{Mb}$ region from 546,086,597 to 547,273,657 bp on the chromosome 5D.

Among the 11 high confidence genes that carry significantly associated SNPs for SBWMV resistance, TraesCS5D01G530600 harbors 12 SNPs including nine new SNPs and three previously reported SNPs (wsnp_JD_ c4438_5567972,wsnp_JD_c4438_5568170 and wsnp_JD_c4438_5567834) ${ }^{17}$. TraesCS5D01G532100 carries five SNPs with four new SNPs. All the remaining SNPs were distributed in nine other genes.

Linkage mapping. To validate the marker-trait association, one SNP was selected from each of the 12 genes harboring the identified SNPs in the association mapping study for KASP assay conversion. The SNPs wsnp_JD_c4438_5568170 from the gene TraesCS5D01G530600 and wsnp_CAP11_c209_198467 from the gene TraesCS5D01G532100 (Table 1) were used to represent the two genes identified in the assays of $90 \mathrm{~K}$ wheat SNP chips. The 12 newly designed KASP-SNPs were used to screen the three parents, Deliver, Wesley, and OK038255403-6 for polymorphism, and ten of the newly designed KASP-SNPs were polymorphic between Wesley and OK03825-5403-6 and eight were polymorphic between Deliver and OK03825-5403-6, respectively. Those polymorphic SNPs also segregated in the two RIL populations.

BLASTN of the 35 marker sequences harboring the significant SNPs against Ae. tauschii physical map identified 14 SNP markers mapped in the physical map (Supplementary Table S4). Ten of the 14 SNPs were successfully converted into KASP assays, but only one SNP (Contig08110_553) showed polymorphism between the two pairs of parents and segregated in the two RIL populations. Together with previously designed 10 and 8 KASP-SNPs in the two populations, a total of 11 and 9 KASP-SNPs linked to SBWMV resistance were mapped on chromosome 


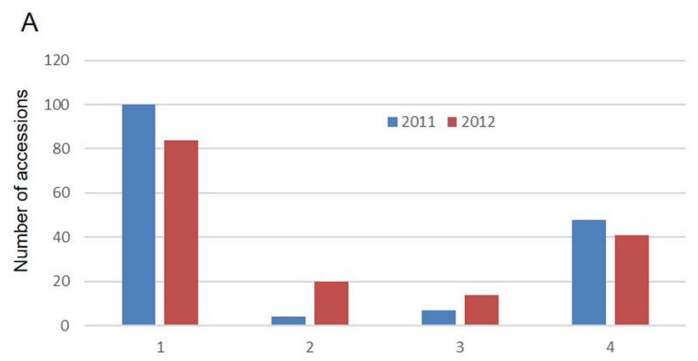

SBWMV score

B
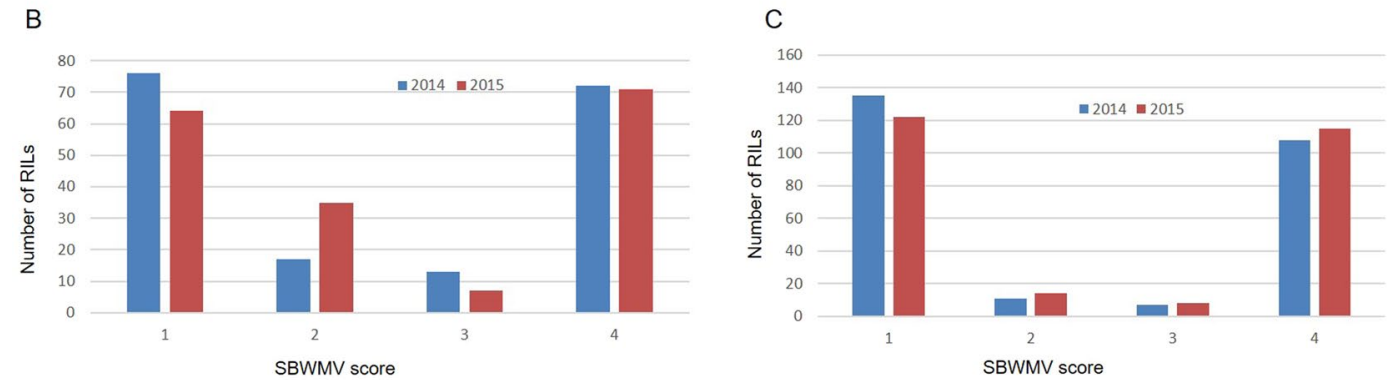

Figure 1. Frequency distribution of Soil-borne wheat mosaic virus disease scores in wheat accessions and recombinant inbred lines (RILs) evaluated in the field experiments at Manhattan, KS. (A) Accessions used for the KASP validation were phenotyped in the spring 2011 and 2012 field experiments; (B) RILs from Wesley $\mathrm{x}$ OK03825-5403-6 $\mathrm{F}_{6}$ population and (C) RILs from Deliver $\mathrm{x}$ OK03825-5403-6 population were phenotyped in the spring 2014 and 2015 field experiments. $1=$ resistant, $2=$ moderately resistant, $3=$ moderately susceptible and $4=$ susceptible.

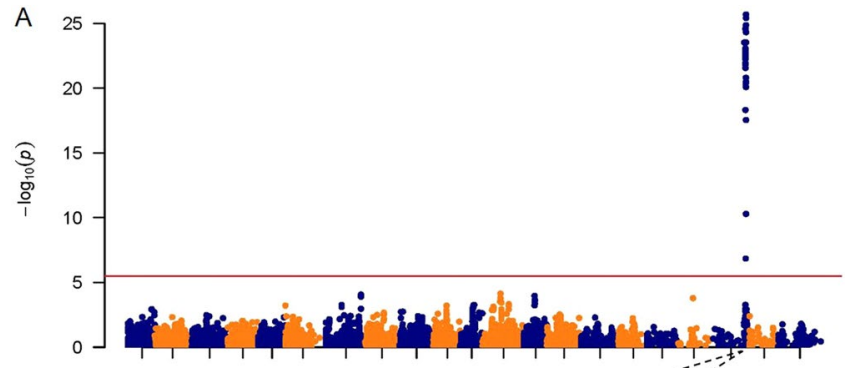

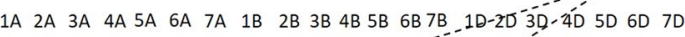

B

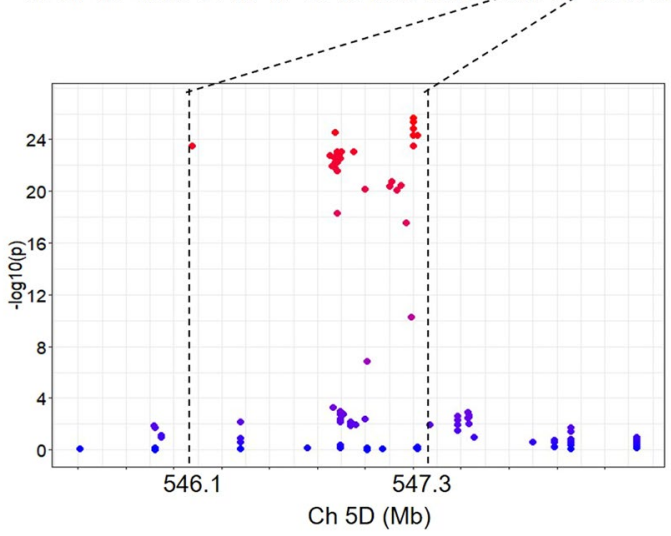

Figure 2. Manhattan plots for wheat resistance to Soil-borne wheat mosaic virus (SBWMV) in an association mapping population (A) and the physical positions of the significant SNPs on chromosome 5D (B). Red line represents the threshold to claim significant SNPs. Two-seasons of SBWMV ratings were collected from two field experiments plus the mean SBWMV ratings over both experiments. 


\begin{tabular}{|c|c|c|c|c|}
\hline SNP Name & SNP Position & MAF $^{\mathbf{a}}$ & Orthologous gene & FDR $^{\mathrm{b}}$ \\
\hline BS00013935_51 & 5D.480829545 & 0.35 & Intergenic region & $8.55 \mathrm{E}-5$ \\
\hline BobWhite_c13030_406 & 5D.549670218 & 0.26 & TraesCS5D01G529700 & $7.89 \mathrm{E}-21$ \\
\hline CAP12_c5949_104 & 5D.550233279 & 0.31 & TraesCS5D01G530200 & $2.16 \mathrm{E}-20$ \\
\hline BS00079676_51 & 5D.550234521 & 0.33 & TraesCS5D01G530300 & $1.05 \mathrm{E}-19$ \\
\hline D_GA8KES401AL4GG_122 & 5D.550235948 & 0.31 & TraesCS5D01G530300 & 2.93E- 20 \\
\hline RAC875_c13169_459 & 5D.550235521 & 0.31 & TraesCS5D01G530300 & $5.32 \mathrm{E}-20$ \\
\hline BS00105939_51 & 5D.550235127 & 0.30 & TraesCS5D01G530400 & $1.28 \mathrm{E}-19$ \\
\hline BobWhite_c4438_162 & 5D.550272236 & 0.32 & TraesCS5D01G530600 & $4.72 \mathrm{E}-20$ \\
\hline BS00088587_51 & 5D.550272456 & 0.31 & TraesCS5D01G530600 & $2.34-20$ \\
\hline BS00088592_51 & 5D.550272362 & 0.37 & TraesCS5D01G530600 & $3.15 \mathrm{E}-16$ \\
\hline Excalibur_c687_886 & 5D.550271002 & 0.29 & TraesCS5D01G530600 & $1.40 \mathrm{E}-21$ \\
\hline Excalibur_c687_907 & 5D.550271023 & 0.31 & TraesCS5D01G530600 & $2.00 \mathrm{E}-20$ \\
\hline Excalibur_c687_961 & 5D.550271077 & 0.31 & TraesCS5D01G530600 & 3.91E-20 \\
\hline IACX10520 & 5D.550272436 & 0.31 & TraesCS5D01G530600 & $2.00 \mathrm{E}-20$ \\
\hline Kukri_c1073_91 & 5D.550271228 & 0.31 & TraesCS5D01G530600 & $1.74 \mathrm{E}-20$ \\
\hline Kukri_c7786_81 & 5D.550241671 & 0.33 & TraesCS5D01G530600 & $2.22 \mathrm{E}-19$ \\
\hline wsnp_JD_c4438_5567834 & 5D.550272167 & 0.31 & TraesCS5D01G530600 & $2.00 \mathrm{E}-20$ \\
\hline wsnp_JD_c4438_5567972 & 5D.550272029 & 0.31 & TraesCS5D01G530600 & $2.39 \mathrm{E}-20$ \\
\hline wsnp_JD_c4438_5568170 & 5D.550271831 & 0.31 & TraesCS5D01G530600 & $5.22 \mathrm{E}-20$ \\
\hline D_GDS7LZN01CBWNE_99 & 5D.550282662 & 0.31 & TraesCS5D01G530700 & $2.92 \mathrm{E}-20$ \\
\hline Kukri_c5528_603 & 5D.550285051 & 0.31 & TraesCS5D01G530700 & $1.74 \mathrm{E}-20$ \\
\hline BS00073116_51 & 5D.550445864 & 0.35 & TraesCS5D01G531100 & $4.36 \mathrm{E}-18$ \\
\hline BS00011469_51 & 5D.550488971 & 0.34 & TraesCS5D01G531200 & $1.24 \mathrm{E}-18$ \\
\hline Excalibur_c14043_548 & 5D.550491920 & 0.38 & TraesCS5D01G531200 & $1.82 \mathrm{E}-15$ \\
\hline Excalibur_c28592_173 & 5D.550491839 & 0.36 & TraesCS5D01G531200 & $2.35 \mathrm{E}-18$ \\
\hline Excalibur_c28592_377 & 5D.550491410 & 0.36 & TraesCS5D01G531200 & 5.53E- 18 \\
\hline Excalibur_c42190_383 & 5D.550487798 & 0.36 & TraesCS5D01G531200 & $2.96 \mathrm{E}-18$ \\
\hline Excalibur_c2795_1518 & 5D.550503097 & 0.20 & TraesCS5D01G531300 & 3.15E- 08 \\
\hline BS00067308_51 & 5D.550837863 & 0.32 & TraesCS5D01G531900 & 9.53E-22 \\
\hline BS00011794_51 & 5D.550854024 & 0.29 & TraesCS5D01G532100 & $4.09 \mathrm{E}-22$ \\
\hline BS00022036_51 & 5D.550854228 & 0.32 & TraesCS5D01G532100 & $1.66 \mathrm{E}-21$ \\
\hline CAP7_rep_c12715_390 & 5D.550853989 & 0.32 & TraesCS5D01G532100 & 7.89E-21 \\
\hline CAP8_c145_89 & 5D.550893835 & 0.30 & TraesCS5D01G532100 & $4.09 \mathrm{E}-22$ \\
\hline wsnp_CAP11_c209_198671 & 5D.550854078 & 0.32 & TraesCS5D01G532100 & $1.66 \mathrm{E}-21$ \\
\hline Excalibur_c22724_85 & 5D.550362064 & 0.31 & TraesCS5D01G625100LC & $1.74 \mathrm{E}-20$ \\
\hline
\end{tabular}

Table 2. Significant SNPs associated with Soil-borne wheat mosaic virus resistance and their corresponding annotated genes. ${ }^{\mathrm{a}}$ Minor allele frequency. ${ }^{\mathrm{b}}$ adjusted $\mathrm{P}$ value.

5D and spanned 11.6cM and 15.4 cM in Wesley $\mathrm{x}$ OK03825-5403-6 and Deliver $\mathrm{x}$ OK03825-5403-6 populations, respectively (Fig. 3). The two flanking markers, wsnp_CAP11_c209_198467 and BS0000079676_51 delimited the gene for SBWMV resistance to $5.1 \mathrm{cM}$ and $3.4 \mathrm{cM}$ intervals in the populations Wesley x OK03825-5403-6 and Deliver x OK03825-5403-6, respectively. The two flanking SNPs represent the two genes that are the closest to Sbwm $1^{17}$ in the two populations, thus, the resistance gene in Wesley and Deliver was most likely the same as Sbwm1 (Fig. 3A).

Putative candidate genes in the genomic region of Sbwm1. BLASTN of the sequences of the two flanking markers, wsnp_CAP11_c209_198467 and BS0000079676_51, for Sbwm1 against the Chinese Spring SeqVer1.0 reference genome identified a $620 \mathrm{~kb}$ interval in wheat on chromosome 5D (Fig. 3). Annotation of the genes in this interval revealed 19 high confidence genes and one low confidence gene (Fig. 3C, Supplementary Table S5). Comparative analysis between wheat and Ae. tauschii, the donor of D genome of hexaploid wheat, identified the conserved collinear orthologous region of $S b w m 1$ with 20 high confidence orthologous genes including the two flanking genes (AET5Gv21176900-AET5Gv21180000) in a $660 \mathrm{~kb}$ interval on the chromosome 5D of Ae. tauschii (Supplementary Table S5). Among the 20 annotated genes of Ae. tauschii, 17 were the same to those in Chinese Spring wheat, and two genes (AET5Gv21177600, AET5Gv21180000) present in Ae. tauschii, but absent in wheat, and one gene (TraesCS5D01G530500) present in wheat, but absent in Ae. tauschii (Fig. 3E). Comparative analysis between wheat and B. distachyon identified a syntenic region (Bradi1g01880 to Bradi1g02020) on chromosome 1 . However, this synteny in B. distachyon was interrupted by four non-syntenic regions (Fig. 3D). Twelve genes were common between B. distachyon and wheat or Ae. tauschii. 


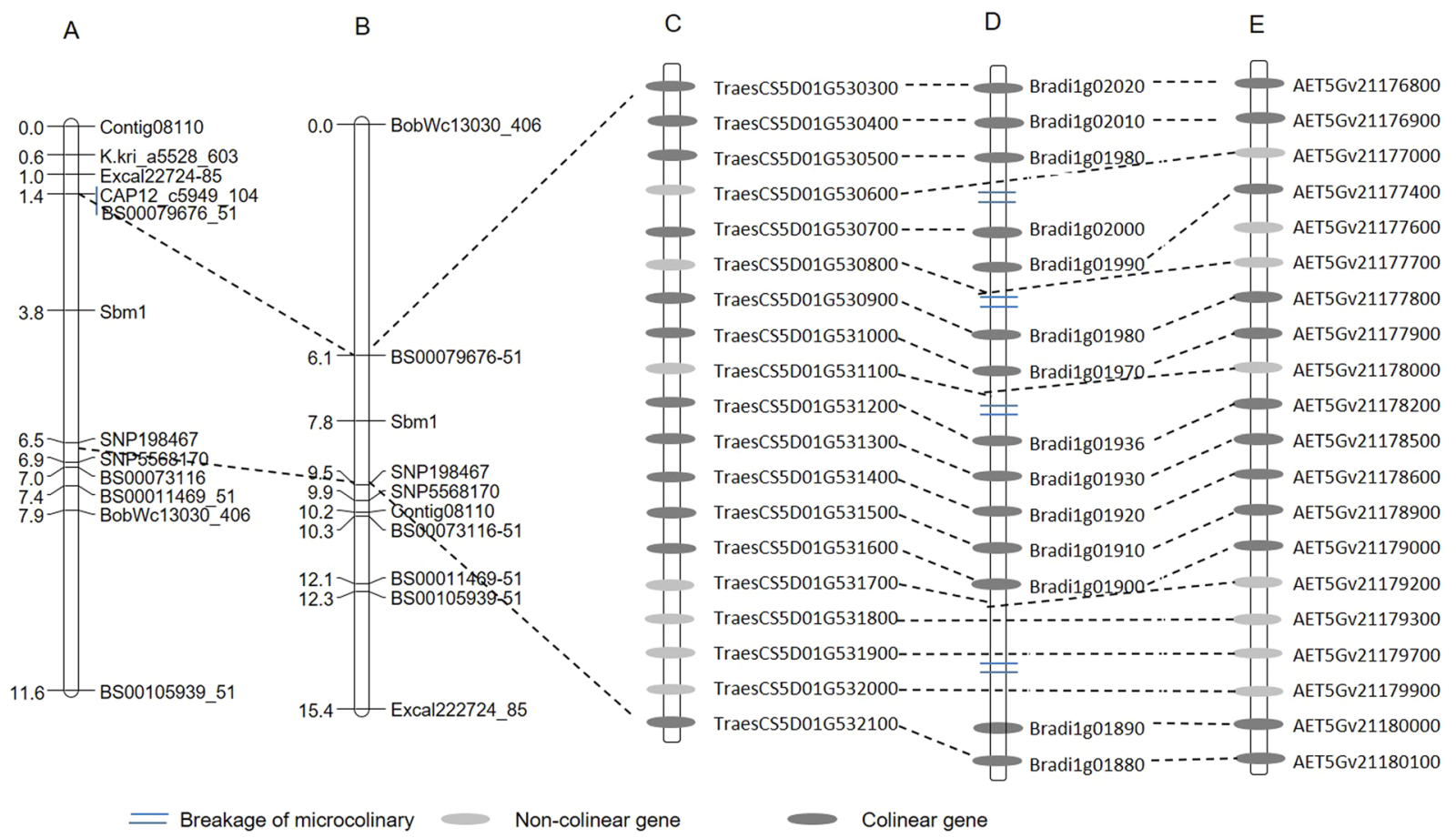

Figure 3. SNP maps of SBWMV-resistance gene (Sbwm1) on chromosome 5D and its syntenic regions in B. distoschyn and Ae. tauschii. (A) A SNP map of Sbwm1 developed from the Wesley x OK03825-5403-6 F 6 population; (B) A SNP map of Sbwm1 developed from the Deliver x OK03825-5403-6 F 6 population; (C) Annotated genes in the $S b w m 1$ region flanked by the two closet markers based on the Chinese Spring reference genome; (D) The syntenic region in B. distachyon with homologous genes; and (E) The syntenic region of Sbwm1 in Ae. tauschii with annotated genes.

Besides the two flanking genes, 17 genes were in the genomic interval annotated from TraesCS5D01G530400 to TraesCS5D01G532000, which include genes encoding a sister chromatid cohesion protein DCC1 (TraesCS5D01G530400), a poly (ADP-ribose) glycohydrolase activity proteins (TraesCS5D01G530500 and TraesCS5D01G530900), a protein kinase family protein (TraesCS5D01G530600), a potassium channel activity protein (TraesCS5D01G530700), a S-adenosylmethionine-dependent methyltransferase (TraesCS5D01G530800), a pentatricopeptide repeat domain family protein (TraesCS5D01G531000), a zinc ion binding protein and zinc finger containing proteins (TraesCS5D01G531100 and TraesCS5D01G531400), a pto-interacting protein 1 (PTI1) (TraesCS5D01G531200), a protein with serine/threonine phosphatase activity (TraesCS5D01G531300), an albino-like protein (TraesCS5D01G531600), a neutral amino acid transmembrane transporter activity protein (TraesCS5D01G531700), a KDEL sequence binding protein (TraesCS5D01G531800), a major pollen allergen-like protein (TraesCS5D01G531900), and a gene encoding a protein with unknown function (TraesCS5D01G531500) (Supplementary Table S3).

Expression test of these genes in leaves showed that eight genes did not express (TraesCS5D01G530400, TraesCS5D01G530400, TraesCS5D01G531400, TraesCS5D01G531500, TraesCS5D01G531700, TraesCS5D01G531900, TraesCS5D01G531200, TraesCS5D01G625100LC), whereas nine expressed but showed the same expression levels between the resistant and susceptible parents (TraesCS5D01G530600, TraesCS5D01G530700, TraesCS5D01G530800, TraesCS5D01G530900, TraesCS5D01G531000, TraesCS5D01G531100, TraesCS5D01G531300, TraesCS5D01G531600, TraesCS5D01G531800) before and after SBWMV symptoms appeared. Only one gene, TraesCS5D01G531200 showed significant differential expression between the resistant and susceptible parents when SBWMV symptoms appeared in the leaf tissues (Fig. 4), indicating that SBWMV infection differentially induced expression of TraesCS5D01G531200. Considering that TraesCS5D01G531200 was the only one of the three genes (the other two were TraesCS5D01G530600 and TraesCS5D01G531300) in this region encoding proteins that may be involved in disease resistance, we believed TraesCS5D01G531200 might play an important role in the SBWMV resistance.

Validation of the flanking markers in cultivars and breeding lines. To further validate the utility of markers that are tightly linked to Sbwm1 in wheat breeding, the two flanking KASP markers, wsnp_CAP11_ c209_198467 and BS0000079676_51, were used to screen 159 additional wheat cultivars and breeding lines from U.S. hard winter wheat breeding programs and the validation panel was also phenotyped for SBWMV resistance in the field experiments (Table 3, Supplementary Table S2). SBWMV resistance of those accessions was consistent between two years with a high correlation of $0.91(p<0.001)$. Among these accessions, 104 were resistant and 55 were susceptible. The two SNPs identified four haplotypes across these accessions (Table 3 ) with the A-A haplotype associated with resistant accessions, and G-G haplotypes with susceptible accessions. For SNP 


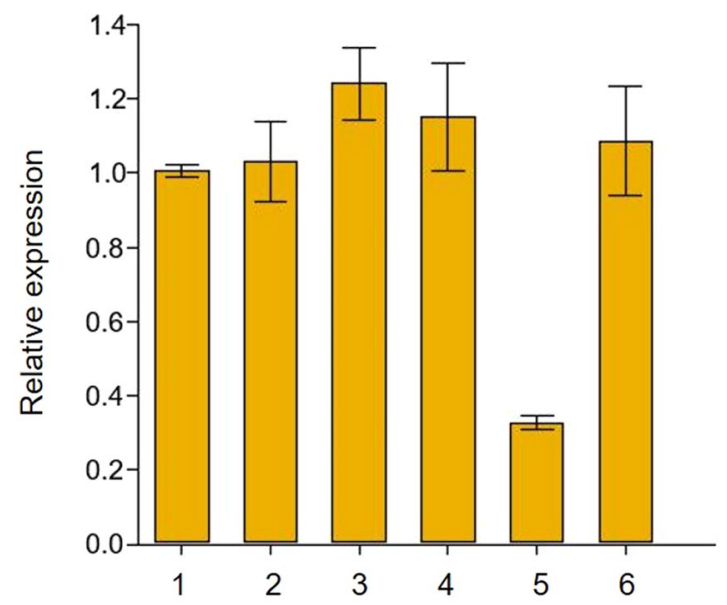

Figure 4. Gene expression analysis of TraesCS5D01G531200 in leaf tissue in the resistant and susceptible parents. Leaf tissue were collected from Wesley (resistant) and OK03825-5403-6 (susceptible) in early spring from uninfected field $(1,2)$, before winter in infected field $(3,4)$, and in early spring from infected field with SBWMV symptom present $(5,6)$. Actin was used as the endogenous control.

\begin{tabular}{|l|l|l|l|}
\hline $\begin{array}{l}\text { wsnp_CAP11_- } \\
\text { c209_198467 }\end{array}$ & B00079676_51 & $\begin{array}{l}\text { Accession } \\
\text { num }\end{array}$ & $\begin{array}{l}\text { SBWMV } \\
\text { resistance }\end{array}$ \\
\hline A & A & 99 & resistant \\
\hline A & G & 5 & resistant \\
\hline G & G & 52 & susceptible \\
\hline G & A & 4 & $\begin{array}{l}\text { resistant (1), } \\
\text { susceptible (3) }\end{array}$ \\
\hline
\end{tabular}

Table 3. Haplotypes and SBWMV reactions of the two flanking SNPs for Sbwm1 in 159 wheat cultivars and breeding lines.

wsnp_CAP11_c209_198467, A presents in 99\% of the resistant accessions and G presents in $98.2 \%$ of susceptible accessions. For SNP BS00079676_51, A and G alleles present in 95.2 and $96.6 \%$ of the resistant and susceptible accessions, respectively. Two recombinant haplotypes A-G and G-A were identified in 5 and 3 accessions, respectively. All the accessions with the A-G haplotype were resistant. For G-A haplotype, however, only one G-A accession was resistant and other two accessions were susceptible, indicating there is recombination between $S b w m 1$ and BS00079676_51 in these two accessions. The two KASP assays together can effectively distinguish resistance and susceptibility alleles in the diverse wheat accessions and can be used for the selection of $S b w m 1$ in breeding programs.

\section{Discussion}

Severity of SBWM infection is affected by environmental factors such as soil water content after planting and temperatures in early spring. In this study, highly repeatable phenotypic data was generated from the two RIL populations, the association mapping population and a validation panel of the 159 wheat accessions in repeated experiments by carefully managing the SBWMV nursery. In the two RIL populations, a slight difference in disease ratings between two years was observed for some RILs (Fig. 1). The rating changes usually occurred within the resistant or susceptible categories, not between the two categories, which can be visualized by high correlation coefficients (from 0.81 to 0.94 ) of SBWMV resistance between years for the four populations investigated. Those results indicated that SBWMV resistance evaluated in the field nursery in this study was highly repeatable, thus is adequate for QTL analysis.

Our previous association mapping study identified an SSR marker, Xgwm469 and two SNPs wsnp_JD_ c4438_5568170,wsnp_CAP11_c209_198467 from 9K SNP chips that closely linked with Sbwm $1^{17,30}$. In the current study, we used the new $90 \mathrm{~K}$ wheat SNP chips with high marker density to conduct a genome-wide association study on the same association mapping panel. We found 21,600 polymorphic SNPs from the $90 \mathrm{~K}$ SNP chips, which is three times of those identified from the $9 \mathrm{~K}$ chips $(6,895 \mathrm{SNP})$. Among the polymorphic SNPs, 35 SNPs were identified to be highly associated with Sbwm1. More trait associated SNPs identified in this study is mainly due to the high SNP density (10 times) in the $90 \mathrm{~K} \mathrm{SNP}$ chip than the $9 \mathrm{~K}$ SNP chip ${ }^{25}$. Among those Sbwm1-associated SNPs, wsnp_JD_c4438_5568170,c4438_5567972,wsnp_JD_c4438_5567834 and wsnp_ CAP11_c209_198671 were the same as previously identified from the 9K SNP chips, and the other 31 were newly identified SNPs from the new $90 \mathrm{~K}$ chips in this study, indicating that increasing marker density facilitate identification of more trait-associated SNPs. Those common SNPs identified in both chips were owing to that the most probes in the $9 \mathrm{~K}$ chip were included in the $90 \mathrm{~K}_{\text {chip }}{ }^{25}$. 
Several independent mapping studies have identified a major locus for SBWMV resistance in the similar location on the long arm of chromosome 5D from different wheat cultivars and an Ae. tauschii-derived breeding line ${ }^{12,16,18}$. Those QTLs most likely underline the same gene, Sbwm1 as reported by Liu et al. ${ }^{17}$. A gene for SBCMV resistance, designated as $S b m 1^{19}$, was also mapped in the similar location as $S b w m 1$ in wheat and they may be either tightly linked or the same gene.

In the linkage maps from Wesley/OK03825-5403-6 and Deliver/OK03825-5403-6 populations, those significant SNPs identified from the AM panel were all mapped near the SBWMV resistance gene in the same linkage group corresponding to the distal end of chromosome 5DL (Fig. 3). The SBWMV resistance gene was flanked by BS00079676_51 and wsnp_CAP11_c209_198467 with BS00079676_51 as the closest marker in the two populations, respectively. Since wsnp_CAP11_c209_198467 has been identified as the closest marker to Sbwm1 in cultivar Heyne ${ }^{17}$, the resistance gene in Wesley and Deliver is in the same region of Sbwm1 and they are most likely the same gene. Therefore, this study defined Sbwm1 region to a 3.4 cM region in the Deliver/OK03825-5403-6 population and identified closely linked flanking markers for $S b w m 1$, which laid solid ground for map-based cloning and marker-assisted breeding of $S b w m 1$. Using association mapping together with linkage mapping can effectively determine the marker-trait association.

Using the IWGSC Chinese Spring reference genome RefSeqv1.0, all the newly identified SNPs were mapped in the interval between 546,086,597 and 547,273,657 on 5D. However, the two flanking SNPs further delimited the $3.4 \mathrm{cM} \mathrm{Sbwm} 1$ region to a physical region of $620 \mathrm{~kb}$ (Fig. 3), suggesting $S b w m 1$ is in a high recombination region. In this region, only 17 high confidence genes were annotated in wheat RefSeqv1.0 reference. Using Ae. tauschii reference genome, $S b w m 1$ was delimited to a $660 \mathrm{~kb}$ region on $5 \mathrm{D}$ with 18 annotated genes (Fig. 3C,E). Only one predicted wheat gene, is absent in Ae. tauschii, and two Ae. tauschii genes are absent in wheat (Supplementary Table S5), indicating good microcolinearity between the two species in Sbmv1 region. In the Sbwm1 genomic region, we identified three candidate genes that are involved in disease resistance. Gene expression analysis identified only one gene showed significantly differential expression between the resistant parents and the susceptible parent (Fig. 4) in the tissues with SBWMV symptoms, which is selected as the candidate for further function analysis.

Wheat SNP chip may not be cost effective for breeding selection due to high cost per sample if only a few SNPs are interested. KASP assay is a time saving and cost-effective genotyping assay for single SNP analysis and has been successfully applied in wheat research and breeding ${ }^{42-44}$. In this study, all the identified SNPs that were highly associated with $S b w m 1$ were converted into KASP assays to map Sbwm1 in the two RIL populations. The KASP assays for those SNPs developed in this study will facilitate isolation and marker-assisted selection of Sbwm1 in wheat.

The effectiveness of the two flanking KASP assays wsnp_CAP11_c209_198467 and BS00079676_51 were also tested in another panel of 159 wheat cultivars and breeding lines. The result showed that when an accession carries the two SNPs in coupling phase, the accession consistently showed expected (resistant or susceptible) phenotypes. However, six accessions carry the two SNPs in repulsion phase and inconsistent phenotypes were found for each recombinant haplotype. When allele A (resistance allele) at wsnp_CAP11_c209_198467 and allele G (susceptibility allele) at BS00079676_51 were together, all accessions with this haplotype showed SBWMV resistance; whereas when accessions carry G (susceptibility allele) at wsnp_CAP11_c209_198467 and A at BS00079676_51 (resistance allele), their reactions to SBWMV infection were mixed (Table 3), indicating that Sbwm 1 located between the two SNPs, and the two SNP together can separate the resistant and susceptible genotypes effectively and can be widely used in marker-assisted breeding to improve SBWMV resistance.

Received: 13 September 2019; Accepted: 20 April 2020;

Published online: 15 May 2020

\section{References}

1. McKinney, H. H. Investigations on the rosette disease of wheat and its control. J. Agric. Res. 23, 771-800 (1923).

2. Barbosa, M., Goulart, L., Prestes, A. \& Juliatti, F. Genetic control of resistance to Soil-borne wheat mosaic virus in Brazilian cultivars of Triticum aestivum L. Thell. Euphytica 122, 417-422 (2001).

3. Hariri, D., Courtillot, M., Zaoui, P. \& Lapierre, H. Multiplication of Soil-borne wheat mosaic virus (SBWMV) in wheat roots infected by a soil carrying SBWMV and wheat yellow mosaic virus (WYMV). Agronomie 7, 789-796 (1987).

4. Kapooria, R. G., Ndunguru, J. \& Clover, G. R. G. First reports of Soil-borne wheat mosaic virus and Wheat spindle streak mosaic virus in Africa. Plant Dis. 84, 921 (2000).

5. Koenig, R. \& Huth, W. Soil-borne rye mosaic and European wheat mosaic virus: two names for a furovirus with variable genome properties which are widely distributed in several cereal crops in Europe. Arch. Virol. 145, 689-697 (2000).

6. Lebas, B. S. M. et al. Investigation of an outbreak of Soil-borne wheat mosaic virus in New Zealand. Aust. Plant Pathol. 38, 85-90 (2009).

7. Sawada, E. Control of Wheat yellow mosaic virus. J. Plant Prot. 14, 444-449 (1927).

8. Driskel, B. A., Hunger, R. M., Payton, M. E. \& Verchot-Lubicz, J. Response of hard red winter wheat to Soil-borne wheat mosaic virus using novel inoculation methods. Phytopathology 92, 347-54 (2002).

9. Shirako, Y., Suzuki, N. \& French, R. C. Similarity and divergence among viruses in the genus Furovirus. Virol. 270, 201-207 (2000).

10. Cadle-Davidson, L., Sorrells, M. E., Gray, S. M. \& Bergstrom, G. C. Identification of small grains genotypes resistant to Soil-borne mosaic virus. Plant Dis. 90, 1039-1044 (2006).

11. Bever, W. M. \& Pendleton, J. W. The effect of Soil-borne wheat mosaic on yield of winter wheat. Plant Dis. Rep. 38, 266-267 (1954).

12. Hao, Y. et al. A conserved locus conditioning Soil-borne wheat mosaic virus resistance on the long arm of chromosome 5D in common wheat. Mol. Breed. 30, 1453-1464 (2012).

13. Myers, L. D., Sherwood, J. L., Siegerist, W. C. \& Hunger, R. M. Temperature-influenced virus movement in expression of resistance to Soil-borne wheat mosaic virus in hard red winter wheat (Triticum aestivum). Phytopathology 83, 548-551 (1993).

14. Merkle, O. G. \& Smith, E. L. Inheritance of resistance to Soil-borne mosaic in wheat. Crop Sci. 23, 1075-1076 (1983).

15. Modawi, R. S., Heyne, E. G., Brunetta, D. \& Willis, W. G. Genetic studies of field reaction to wheat Soil-borne mosaic virus. Plant Dis. 66, 1183-1184 (1982) 
16. Narasimhamoorthy, B., Gill, B. S., Fritz, A. K., Nelson, J. C. \& Brown- Guedira, G. L. Advanced backcross QTL analysis of a hard winter wheat $\mathrm{x}$ synthetic wheat population. Theor. Appl. Genet. 112, 787-796 (2006).

17. Liu, S., Yang, X., Chao, S., Bockus, W. \& Bai, G. Genome-wide association analysis identified a major QTL conferring wheat Soilborne wheat mosaic virus resistance. Theor. Appl. Genet. 127, 1039-1047 (2014).

18. Hall, M. D., Brown-Guedira, G., Klatt, A. \& Fritz, A. K. Genetic analysis of resistance to Soil-borne wheat mosaic virus derived from Aegilops tauschii. Euphytica 169, 169-176 (2009).

19. Bass, C., Hendley, R., Adams, M. J., Hammond-Kosack, K. E. \& Kanyuka, K. The Sbm1 locus conferring resistance to Soil-borne cereal mosaic virus maps to a gene-rich region on 5DL in wheat. Genome 2006(49), 1140-8 (2006).

20. Perovic, D. et al. Mapping and diagnostic marker development for Soil-borne cereal mosaic virus resistance in bread wheat. Mol. Breed. 23, 641-653 (2009).

21. Manish, K. P. et al. Development and evaluation of a high density genotyping 'Axiom_Arachis' array with 58 K SNPs for accelerating genetics and breeding in groundnut. Sci. Reports 7, 40577, https://doi.org/10.1038/srep40577 (2017).

22. Huang, X. et al. Genome-wide association studies of 14 agronomic traits in rice landraces. Nat. Genet. 42, 961-967 (2010).

23. Belamkar, V. et al. Genomic selection in preliminary yield trials in a winter wheat breeding program. G3(8), 2735-2747 (2018).

24. Cavanagh, C. R. et al. Genome-wide comparative diversity uncovers multiple targets of selection for improvement in hexaploid wheat landraces and cultivars. Proc. Natl. Acad. Sci. USA 110, 8057-8062 (2013).

25. Wang, S. et al. Characterization of polyploid wheat genomic diversity using a high-density 90,000 SNP array. Plant Biotech. J. 12, 787-96 (2014).

26. Winfield, M. Q. et al. High-density SNP genotyping array for hexaploid wheat and its secondary and tertiary gene pool. Plant Biotechnol. J. 14, 1195-1206 (2016).

27. Rasheed, A. et al. Crop breeding chips and genotyping platforms: progress, challenges, and perspectives. Mol. Plant 10, 1047-1064 (2017).

28. Allen, A. M. et al. Characterization of a Wheat Breeders' Array suitable for high-throughput SNP genotyping of global accessions of hexaploid bread wheat (Triticum aestivum). Plant Biotech. J. 15, 390-401 (2017).

29. Zhang, D., Bai, G., Zhu, C., Yu, J. \& Carver, B. F. Genetic diversity, population structure, and linkage disequilibrium in U.S. elite winter wheat. The Plant Genome 3, 117-127 (2010).

30. Zhang, D. et al. Association study of resistance to Soil-borne wheat mosaic virus in U.S. winter wheat. Phytopathology 101, 1322-9 (2011).

31. Lin, M. et al. Genome-wide association analysis on pre-harvest sprouting resistance and grain color in U.S. winter wheat. $B M C$ Genomics 17, 794 (2016).

32. Rudi, A. et al. Shifting the limits in wheat research and breeding using a fully annotated reference genome. Science 361, 6403 (2018).

33. Luo, M. C. et al. A 4-gigabase physical map unlocks the structure and evolution of the complex genome of Aegilops tauschii, the wheat D-genome progenitor. Proc. Natl. Acad. Sci. USA 110, 7940-7945 (2013).

34. Toojinda, T., Baird, E., Booth, A., Broers, L. \& Hayes, P. Introgression of quantitative trait loci (QTLs) determining stripe rust resistance in barley: an example of marker assisted line development. Theor. Appl. Genet. 96, 123-131 (1998).

35. Pritchard, J. K., Stephens, M. \& Donnelly, P. Inference of population structure using multi-locus genotype data. Genetics 155, 945-59 (2000).

36. Hardy, O. J. \& Vekemans, X. Spagedi: a versatile computer program to analyze spatial genetic structure at the individual or population levels. Mol. Ecol. Notes 2, 618-20 (2002).

37. Lipka, A. E. et al. GAPIT: genome association and prediction integrated tool. Bioinformatics 28, 2397-9 (2012).

38. Van Ooijen, J. W. \& Voorrips, R. E. JionMap version 3.0: Software for the calculation of genetic linkage maps (2001).

39. Kosambi, D. D. The estimation of map distances from recombination values. Ann. Eugen. 12, 172-175 (1944).

40. Luo, M. et al. Genome sequence of the progenitor of the wheat D genome Aegilops tauschii. Nature 551, 498-502 (2018).

41. The International Brachypodium Initiative. Genome sequencing and analysis of the model grass Brachypodium distachyon. Nature 463, 63-768 (2010)

42. Terracciano, I. et al. Development of COS-SNP and HRM markers for high throughput and reliable haplotype-based detection of Lr14a in durum wheat (Triticum durum Desf.). Theor. Appl. Genet. 126, 1077-1101 (2013).

43. Semagn, K., Babu, R., Hearne, S. \& Olsen, M. Single nucleotide polymorphism genotyping using Kompetitive Allele Specific PCR (KASP): overview of the technology and its application in crop improvement. Mol. Breed. 33, 1-14 (2014).

44. Rasheed, A. et al. Development and validation of KASP assays for genes underpinning key economic traits in bread wheat. Theor. Appl. Genet. 129, 1843-1860 (2016)

\section{Acknowledgements}

This project is partly funded by the National Key Research and Development Programs of China (2016YFD0101802, 2016ZX08009003-001-006), National Research Initiative Competitive Grants 2017-6700725939, and 2017-67007-25929 from the USDA National Institute of Food and Agriculture, and the Shandong Province Agricultural Fine Seeds Project (2016LZGC023). Mention of trade names or commercial products in this article is solely for the purpose of providing specific information and does not imply recommendation or endorsement by the U.S. Department of Agriculture. USDA is an equal opportunity provider and employer.

\section{Author contributions}

S.L. and G.B. wrote the manuscript; S.L., F.J. and D.Z. performed field evaluation and genotyping; S.L., M.L. and G.B. analyzed the data; M.L. contributed to the marker development; B.T., H.N.T. and L.Y. contributed to the writing of the manuscript.

\section{Competing interests}

The authors declare no competing interests.

\section{Additional information}

Supplementary information is available for this paper at https://doi.org/10.1038/s41598-020-64993-3.

Correspondence and requests for materials should be addressed to S.L. or G.B.

Reprints and permissions information is available at www.nature.com/reprints.

Publisher's note Springer Nature remains neutral with regard to jurisdictional claims in published maps and institutional affiliations. 
(c) (i) Open Access This article is licensed under a Creative Commons Attribution 4.0 International License, which permits use, sharing, adaptation, distribution and reproduction in any medium or format, as long as you give appropriate credit to the original author(s) and the source, provide a link to the Creative Commons license, and indicate if changes were made. The images or other third party material in this article are included in the article's Creative Commons license, unless indicated otherwise in a credit line to the material. If material is not included in the article's Creative Commons license and your intended use is not permitted by statutory regulation or exceeds the permitted use, you will need to obtain permission directly from the copyright holder. To view a copy of this license, visit http://creativecommons.org/licenses/by/4.0/.

(C) The Author(s) 2020 